\title{
Quantile-Based Estimative VaR Forecast and Dependence Measure: A Simulation Approach
}

\author{
Khreshna Syuhada $(\mathbb{D}$, Risti Nur'aini, and Mahfudhotin \\ Statistics Research Division, Institut Teknologi Bandung, Jalan Ganesa 10, Bandung 40132, Indonesia \\ Correspondence should be addressed to Khreshna Syuhada; khreshna@math.itb.ac.id
}

Received 28 September 2019; Revised 21 January 2020; Accepted 24 February 2020; Published 23 April 2020

Academic Editor: Qingbiao Wu

Copyright (c) 2020 Khreshna Syuhada et al. This is an open access article distributed under the Creative Commons Attribution License, which permits unrestricted use, distribution, and reproduction in any medium, provided the original work is properly cited.

\begin{abstract}
A Value-at-Risk (VaR) forecast may be calculated for the case of a random loss alone and/or of a random loss that depends on another random loss. In both cases, the VaR forecast is obtained by employing its (conditional) probability distribution of loss data, specifically the quantile of loss distribution. In practice, we have an estimative VaR forecast in which the distribution parameter vector is replaced by its estimator. In this paper, the quantile-based estimative VaR forecast for dependent random losses is explored through a simulation approach. It is found that the estimative VaR forecast is more accurate when a copula is employed. Furthermore, the stronger the dependence of a random loss to the target loss, in linear correlation, the larger/smaller the conditional mean/variance. In any dependence measure, generally, stronger and negative dependence gives a higher forecast. When there is a tail dependence, the use of upper and lower tail dependence provides a better forecast instead of the single correlation coefficient.
\end{abstract}

\section{Introduction}

A Value-at-Risk (VaR) forecast is crucial as a main reference for banking and insurance industries in assessing their financial risk performance as well as in allocating their capital. The VaR forecast may also be used as an alarm for such industries before they reach the worst risk. It is, therefore, a must for a risk manager to have accurate and reliable forecast of VaR.

Forecasting $\mathrm{VaR}$, in general, requires the quantile of the loss distribution (see, e.g., [1-4], for review of VaR forecast); later, we name this as the quantile-based VaR forecast which will be described in Section 2 through simulation. In practice, however, we may obtain $\mathrm{VaR}$ of loss data by simply taking the quantile of its ordered data (known as the historical simulation method) without knowing its estimate of probability function or density. This approach, however, may not be satisfied and has the possibility of high forecast error. Other approaches include variance-covariance and Monte Carlo methods with some restrictions such as normality distribution assumption and accurate parameter estimate. In fact, there are still efforts by authors to develop the best risk forecast models.

A VaR forecast model may typically be developed in the direction of proposing the best $\mathrm{VaR}$ forecast technique along with its accuracy. For example, we may forecast VaR by a technique that substitutes model parameters by their estimates. The resulting $\mathrm{VaR}$ forecast, called an estimative $\mathrm{VaR}$ forecast, has coverage probability accuracy bounded to $O\left(n^{-1}\right)$. To have the best VaR forecast, one then can modify the calculation to yield the so called improved $\mathrm{VaR}$ forecast (e.g., [5-7]). This improved VaR forecast is more accurate and has coverage property bounded to $O\left(n^{-3 / 2}\right)$. An alternative direction of having the best VaR forecast is by incorporating statistical properties of loss distribution in the $\mathrm{VaR}$ forecast technique such as high kurtosis, volatility clustering, and heavy-tailed distribution, e.g., $[8,9]$, or in relation to extreme observations as in [10]. It is hoped that the resulting $\mathrm{VaR}$ forecast is not only having the best accuracy but also capturing the empirical moment as well as distributional properties. 


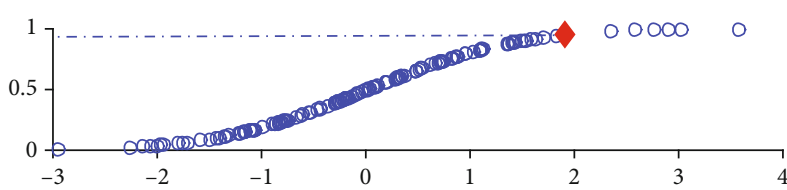

(a)

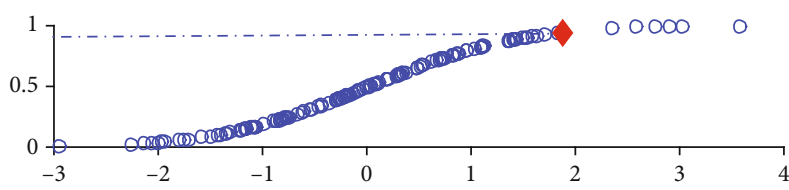

(c)

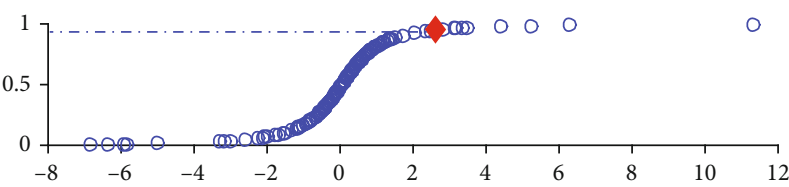

(e)

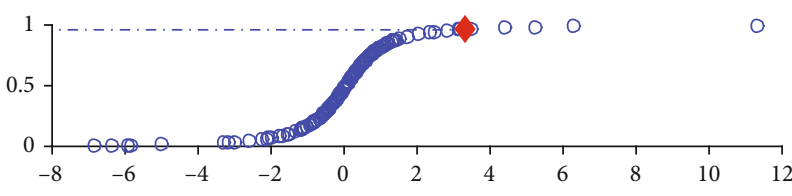

(g)

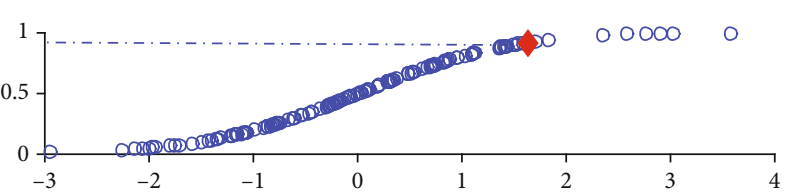

(b)

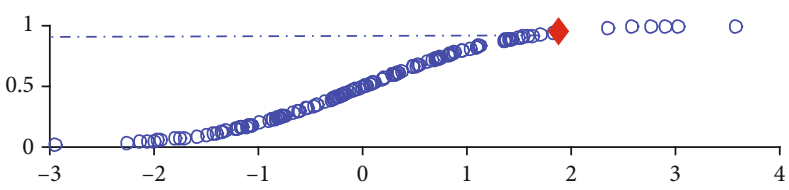

(d)

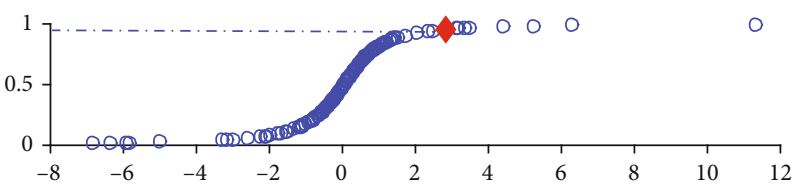

(f)

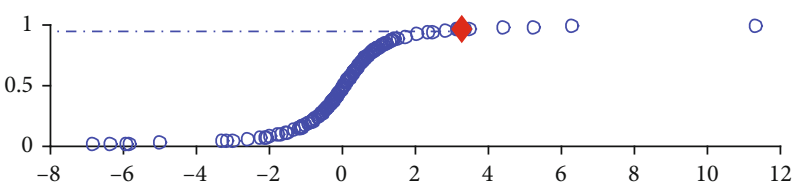

(h)

Figure 1: VaR forecast of normal distribution $(\mathrm{a}-\mathrm{d})$ and $t$ distribution $(\mathrm{e}-\mathrm{h})$ calculated from the distribution function; the red dot mark in the figure shows the "position" of the VaR forecast (to $x$-axis) and its corresponding $(1-\alpha)$ (to $y$-axis).

In this paper, we aim to explore the $\mathrm{VaR}$ forecast in a different direction for a random loss that depends on another random loss, i.e., forecasting VaR for dependent losses. In particular, we are interested in finding the effect of dependence measures, such as unconditional correlation and tail dependence, to the estimative VaR forecast. There have been some works related to the dependence and VaR forecast. For instance, Ku and Wang [11] have used the MGARCH model with dynamic conditional correlation (DCC), instead of constant correlation, in backtesting the $\mathrm{VaR}$ forecast through forecast failures and average deviations. Meanwhile, Santos and Alves [10] considered dependence of excesses and duration by using S\&P 500 index and found that the linear correlation was weak. In addition, Messaoud and Aloui [12] calculated the VaR and CoVaR forecast based on the Clayton copula which is in fact less than those based on the t/normal copula since the Clayton copula has tail correlation. Multivariate $t$ copula shows more observations in the tails, a function of correlation.

To begin with, we review, through examples and simulation, the quantile-based VaR forecast for a random loss alone and/or a random loss that depends on another random loss, in Section 2. We visualize such estimative VaR forecasts along with their coverage probability via distribution function and probability function. It is important to note that the use of a copula to obtain bivariate distribution function is unavoidable due to nonnormality or nonidenticality of marginal loss distributions.

In Section 3, we present the conditional estimative $\mathrm{VaR}$ forecast that is explicitly formed as the conditional
TABLE 1: The estimative VaR forecast and its coverage probability (in the bracket) of a random loss.

\begin{tabular}{lcc}
\hline & Normal & $t$ \\
\hline Quantile-based & $1.7182(0.9500)$ & $2.3341(0.9500)$ \\
Historical simulation & $1.6946(0.9476)$ & $2.1947(0.9442)$ \\
Monte Carlo & $1.7182(0.9500)$ & $2.6182(0.9596)$ \\
Variance-covariance & $1.7070(0.9489)$ & $2.8759(0.9663)$ \\
\hline
\end{tabular}

mean and variance; we take the case symmetrical distributions of normal and $t$ random samples. The effect of some dependence measures such as unconditional correlation and tail dependence is shown. Furthermore, we describe, in Section 4, dependent random losses for a certain stochastic process, namely, Autoregressive Conditional Heteroscedasticity (ARCH); note that the Generalized ARCH model, known as GARCH, has been also the interest for authors, including [2], in finding the VaR forecast with a copula. For this stochastic process-type dependent random loss case, we treat it as from (i) a single stochastic process and (ii) two dependent stochastic processes. Finally, Section 5 considers the dependent random losses with upper tail dependence coefficient. We show and compare simulated data to find the estimative $\mathrm{VaR}$ forecast by involving the tail dependence coefficient in comparison to a single correlation coefficient or two correlation coefficients. To illustrate our proposed method on involving dependence for the VaR forecast, we simulate such calculations for real data of the S\&P 500 index and the Dow Jones index; this is described in Section 6. The discussion is given in Section 7. 


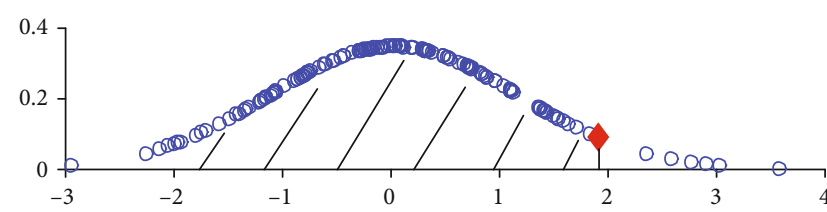

(a)

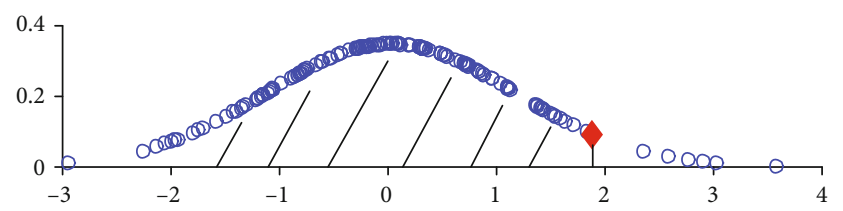

(c)

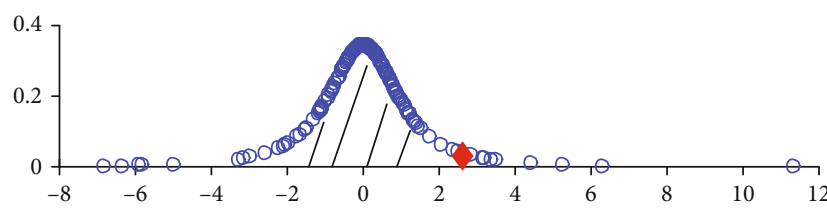

(e)

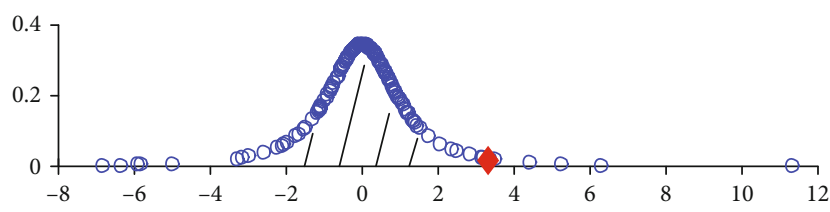

(g)

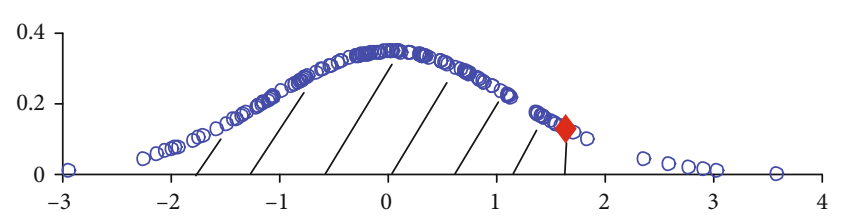

(b)

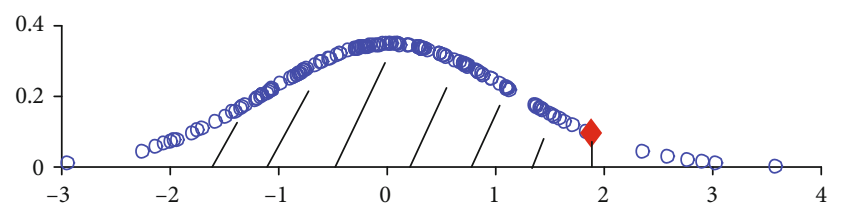

(d)

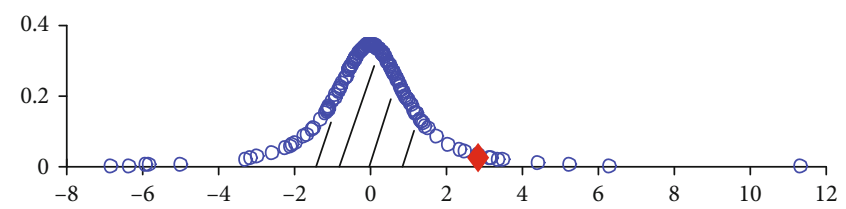

(f)

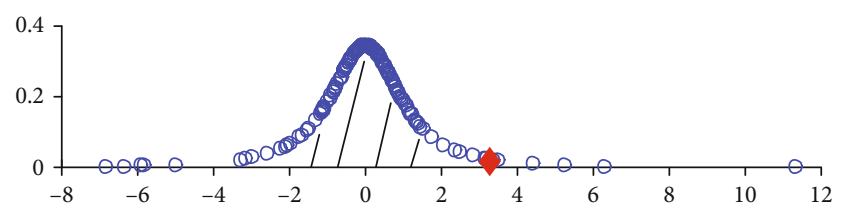

(h)

FIGURE 2: The corresponding probability function-based visualization of calculation for the VaR forecast of normal and $t$ distributions; the red dot mark in the figure shows the "position" of the VaR forecast (to $x$-axis) and its corresponding $(1-\alpha)$ (under the line).

\section{Motivating Examples of Quantile-Based Value-at-Risk Forecast}

From a statistical perspective, the Value-at-Risk (VaR) forecast is an application of the concept of (upper) forecasting limit for future observations, given a collection of random variables for losses. Suppose that a random loss $X$ has distribution function $F_{X ; \theta}$, where $\theta$ is the distribution parameter. Provided that the inverse of distribution function exists, we obtain $\operatorname{VaR}$ of $X$ at the level of significance $\alpha$ :

$$
\operatorname{VaR}_{\alpha}(X ; \theta)=F_{X ; \theta}^{-1}(1-\alpha)
$$

for all $\theta$; for more general random loss, we may use the $\mathrm{VaR}$ forecast formula as $\operatorname{VaR}_{\alpha}(X ; \theta)=\inf \left\{x ; F_{X ; \theta}(x) \geq 1-\alpha\right\}$, see, e.g., $[3,13]$. For symmetrical distributions such as normal and $t, F_{X ; \theta}^{-1}(1-\alpha)$ in (1) consists of the mean and variance of $X$. Furthermore, in practice, parameter $\theta$ must be estimated from data. The estimative $\mathrm{VaR}$ forecast, $\operatorname{VaR}_{\alpha}(X$; $\widehat{\theta})$ or $\widehat{\mathrm{VaR}}_{\alpha}(X ; \widehat{\theta})$, is obtained by substituting parameter $\theta$ with its estimate $\widehat{\theta}$ as in $[5,6]$; the common estimators used are the maximum likelihood and least square. As mentioned early in this section, VaR forecast calculation is actually the (upper) forecasting limit of $X_{n+1}$ when the available data are $X_{1}, X_{2}, \cdots, X_{n}$. We may assume that the distribution of $X_{n+1}$ is identical to $X_{i}$, for $i=1,2, \cdots, n$. Note that forecasting VaR may be carried out for the $d$-step-ahead forecast.
We illustrate, in Figure 1, the VaR forecast of normal (Figures $1(\mathrm{a})-1(\mathrm{~d})$ ) and $t$ (Figures $1(\mathrm{e})-1(\mathrm{~h})$ ) distributions. The red dot mark in the figure shows the "position" of the $\mathrm{VaR}$ forecast (to $x$-axis) and its corresponding $(1-\alpha)$ (to $y$-axis). The first row (Figures 1 (a) and $1(\mathrm{e})$ ) is obtained by taking the quantile of such distributions. The next three rows explain the VaR forecast calculated from some approaches of historical simulation (Figures 1(b) and 1(f)), Monte Carlo (Figures 1(c) and 1(g)), and variancecovariance (Figures $1(\mathrm{~d})$ and $1(\mathrm{~h})$ ). It may be observed that the $\mathrm{VaR}$ forecast obtained from the variance-covariance method has an interesting feature: a lower value than other methods (for normal distribution) and a higher value than others for $t$ distribution (see Table 1 for the exact number). The corresponding $\mathrm{VaR}$ forecasts calculated from the probability function are shown in Figures 2(a)-2(d) for normal distribution and Figures $2(\mathrm{e})-2(\mathrm{~h})$ for $t$ distribution. The visualization of the probability function-based VaR forecast instead of distribution function may be easier for some readers as the coverage probability $(1-\alpha)$ for such VaR forecasts is easy to see in the area under the line (see, for instance, [3]), whilst the sample quantile for normal and $t$ distributions along with their bands may be found in [14]. We may conclude that the quantile-based estimative VaR forecasts obtained directly from (normal and $t$ ) loss distributions are more accurate than other methods.

The choice of normal and $t$ distributions for the $\mathrm{VaR}$ forecast illustration above came from the fact that both are symmetrical distribution and location-scale family (thus, 

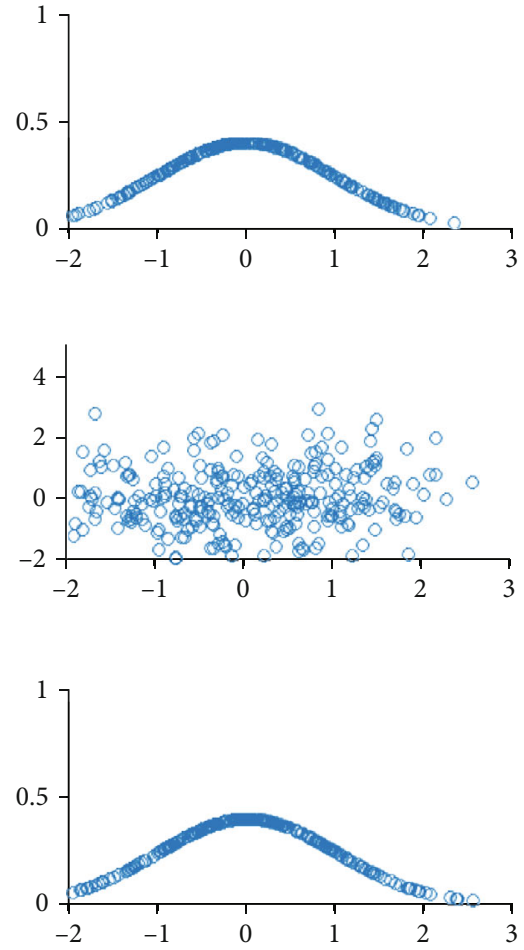

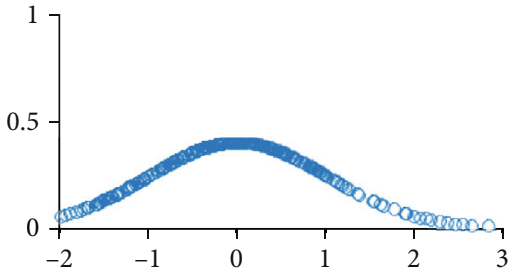

(a)

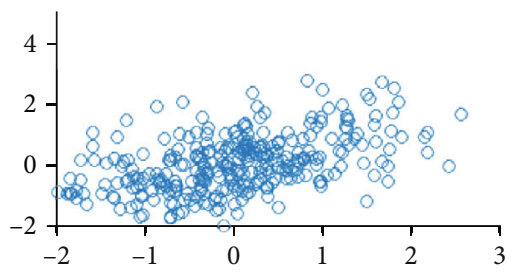

(b)

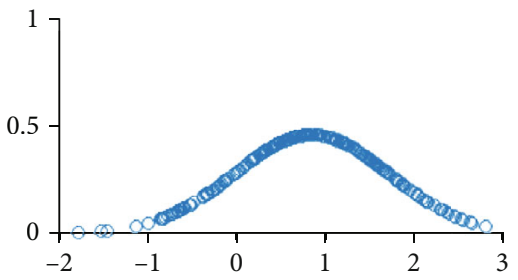

(c)
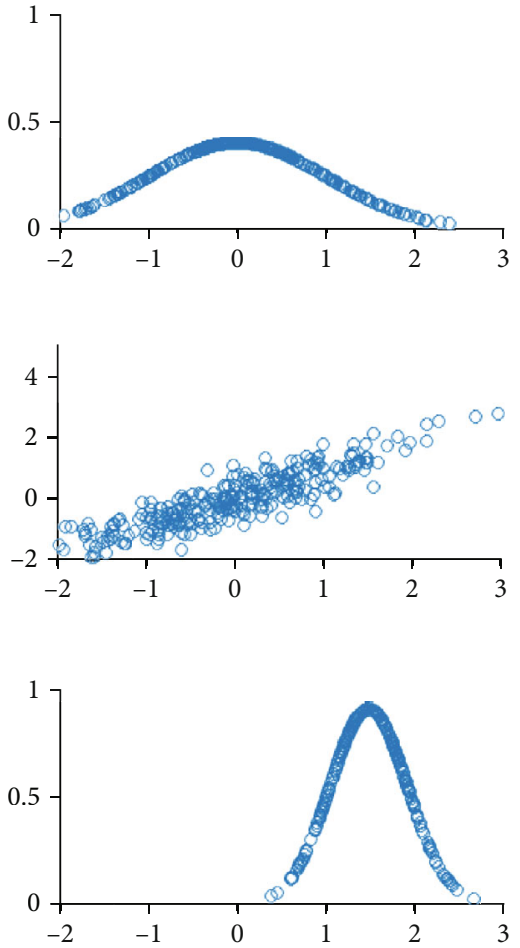

Figure 3: The simulated data of univariate (a) and of conditional (c) normal distributions; (b) displays the corresponding scatter plot of bivariate normal random losses.

they are comparable) with certain features of heavy tail for $t$ distribution. The estimative $\mathrm{VaR}$ forecast of normal distribution with parameter $\theta=(\mu, \sigma 2)$ is

$$
\widehat{\mathrm{VaR}}_{\alpha}(X ; \widehat{\theta})=\widehat{\mu}+\widehat{\sigma} \Phi^{-1}(1-\alpha)
$$

where $\Phi$ and $\Phi^{-1}(1-\alpha)$ denote the distribution function and $(1-\alpha)$-quantile of the standard normal, respectively. Meanwhile, the $\mathrm{VaR}$ forecast for $t$ distribution with degrees of freedom $\kappa, t_{\kappa}$, which has mean $\mu$ and variance $\sigma^{2} \kappa /(\kappa-2)$, for $\kappa>2$, is $\widehat{\mu}+\widehat{\sigma} t_{\kappa}^{-1}(1-\alpha)$, where $t_{\kappa}$ denotes distribution function of standard $t$. The class of (i) (a)symmetrical, (ii) heavy-tailed, and/or (iii) extreme distributions in the VaR forecast, typically for return innovation, has also been discussed by authors, e.g., [8-10, 13].

2.1. The Example of Two Dependent Random Losses. We consider now two random losses where one depends on another random loss. For example, observe bivariate normal distribution simulated data (second and third rows) with several correlation coefficients: $0.1-0.5-0.9$ (first-second-third columns) in Figure 3. To compare with, a univariate normal distribution simulated data is given (Figure 3(a)). It is interesting to learn a quite different probability function of univariate and bivariate normal data and perhaps the effect of dependence.

Suppose that two random losses $X$ and $Y$ are dependent on the parameter vector, for their joint distribution function, $\omega$ and dependence measure $\rho(\omega)$. We assume that their dependence may be a Pearson linear correlation, a rank correlation, and the coefficient of tail dependence, where the last two are copula-based dependence measures [3]. Our aim is to find the $\operatorname{VaR}$ forecast of $X$, given $Y$, such that it has conditional coverage probability equal to $1-\alpha$, i.e.,

$$
P\left(X \leq \operatorname{VaR}_{\alpha}(X \mid Y ; \rho(\omega)) \mid Y\right)=1-\alpha,
$$

for all $\omega$; in other words, the $\mathrm{VaR}$ forecast (3) is obtained by calculating the inverse of conditional distribution function of $X \mid Y, F_{X}{ }^{-1}{ }_{Y ; \omega}(1-\alpha)$. It is, therefore, important to derive a joint distribution function of $(X, Y), F_{X, Y ; \omega}(x, y ; \omega)$, in order to obtain conditional distribution function $\left.F_{X}\right|_{Y ; \omega}$. It is easy to have bivariate normal or $t$ distribution. However, when the distributions of $X$ and $Y$ are neither normal nor identical, we may need a copula to obtain such joint distribution function.

We briefly describe a copula as follows. Let $F_{X}$ and $G_{Y}$ denote the distribution function for $X$ and $Y$, respectively. Defining $U=F_{X}(X)$ and $V=G_{Y}(Y)$, each has uniform distribution in $[0,1]$. According to Sklar's theorem, a twodimensional copula $C:[0,1]^{2} \longrightarrow[0,1]$ is the distribution function $C(u, v)=C\left(F_{X}(x), G_{Y}(y)\right)=H_{X, Y}(x, y)$, where $H_{X, Y}$ is the joint distribution function of $(X, Y)$ (see, e.g., [12, 15]). The copula will be beneficial not only for reducing parameters (particularly in an explicit copula) but also for relaxing dependence models and understanding their dependence more easily [3]. For example, the class of the 


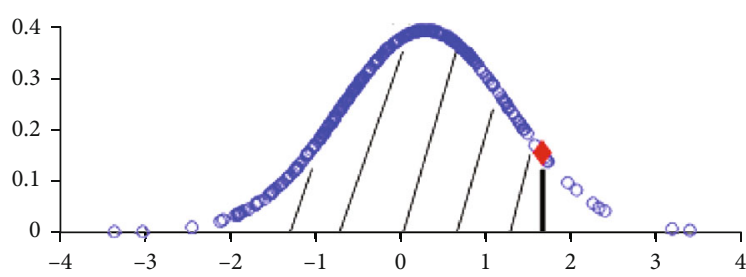

(a)

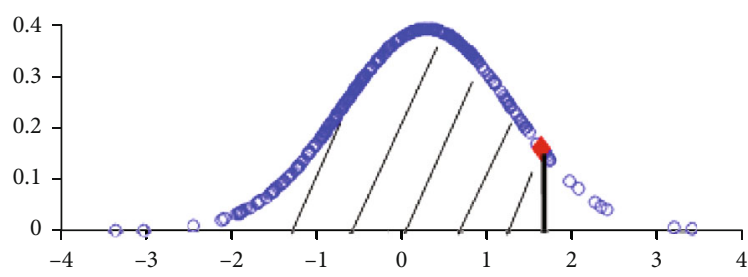

(c)

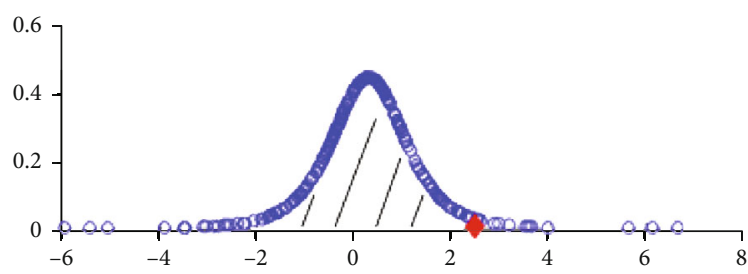

(e)

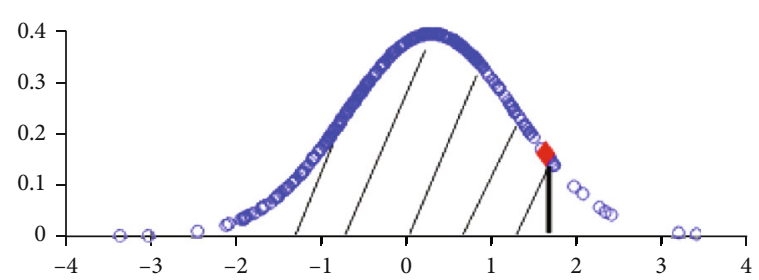

(b)

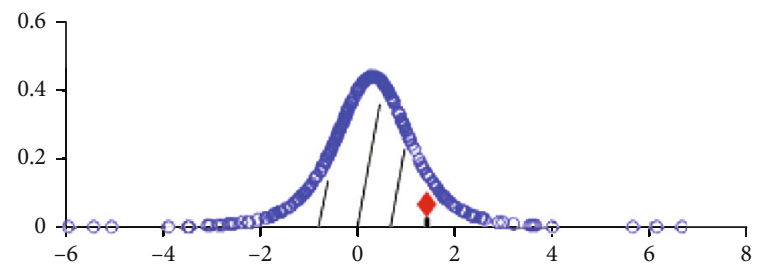

(d)

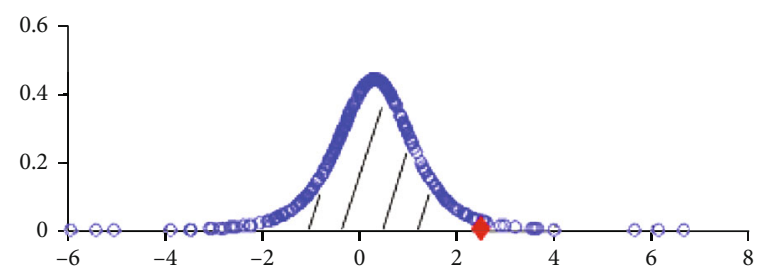

(f)

FIgURE 4: Quantile-based VaR forecast (the red dot mark) of normal (left column) and $t$ (right column) distributed data obtained from bivariate normal (a) and $t(\mathrm{~d})$ distributions. The implicit copulas (Gaussian and $t$ ) are shown in (b) and (e), respectively, whilst the use of Clayton copula (explicit copula, member of Archimedean copula) is shown in (c, f), respectively.

Archimedean copula, namely, Gumbel and Clayton copulas, are

$$
\begin{aligned}
& C^{\mathrm{G}}(u, v)_{\theta}=\exp \left(-\left((-\ln (u))^{\theta}+(-\ln (v))^{\theta}\right)^{1 / \theta}\right), \quad 0 \leq \theta<\infty, \\
& C^{\mathrm{C}}(u, v)_{\theta}=\left(u^{-\theta}+v^{-\theta}-1\right)^{-1 / \theta}, \quad-1<\theta<\infty, \theta \neq 0,
\end{aligned}
$$

which reduce the parameter vector of bivariate normal $\left(\mu_{X}, \mu_{Y}, \sigma_{X}{ }^{2}, \sigma_{Y}^{2}\right)$ to only one parameter $\theta$. The Gumbel copula is more appropriate for data with upper tail dependence whilst the Clayton copula represents phenomena of lower tail dependence. Meanwhile, the implicit copulas of Gaussian and $t$ are not in the closed form and are represented as

$$
\begin{aligned}
C^{\mathrm{Ga}}(u, v)= & \int_{-\infty}^{\Phi^{-1}(u)} \int_{-\infty}^{\Phi^{-1}(v)} \frac{1}{2 \pi\left(1-\rho(\omega)^{2}\right)^{1 / 2}} \exp \\
& \cdot\left(\frac{-\left(r_{1}^{2}-2 \rho(\omega) r_{1} r_{2}+r_{2}^{2}\right)}{2\left(1-\rho(\omega)^{2}\right)}\right) d r_{1} d r_{2},
\end{aligned}
$$

and $C_{\kappa}^{t}(u, v)=t_{\kappa, K}\left(t_{\kappa}^{-1}(u), t_{\kappa}^{-1}(v)\right)$, respectively, where $t_{\kappa, K}$ denotes joint distribution function and $t_{\kappa}$ is the distribution function of standard $t$. In contrast to the Gumbel and Clayton copulas, the Gaussian copula does not have tail dependence. On the other hand, the $t$ copula has both upper and lower tail dependence and is suitable for financial return data [2].

In Figure 4, we present the estimative VaR forecast (in the probability function-based visualization) for normal (left column) and $t$ (right column) distributed data; in Figures 4(a) and $4(\mathrm{~d})$, such forecasts are computed by using the bivariate normal, $H_{X, Y}{ }^{\text {Normal }}(x, y)$, and $t, H_{X, Y}{ }^{t}(x, y)$, distribution functions. The estimative VaR forecast (in the red dot mark) obtained from Gaussian and $t$ copulas (member of implicit copula) is shown in Figures 4(b) and 4(e), respectively; in Figures 4(c) and 4(e), the use of the Clayton copula (which is a member of the Archimedean copula) is presented. As in Table 2 (for the exact number), we find a quite similar result of the estimative $\mathrm{VaR}$ forecast computed by bivariate normal $(t)$ and the corresponding Gaussian $(t)$ copula.

\section{Correlation Dependence and Conditional VaR Forecast: The Case of Symmetrical Distribution}

3.1. Normal Distribution. Let $X$ and $Y$ be two dependent random losses assumed normally distributed. The mean and variance vectors are $\left(\mu_{X}, \mu_{Y}\right)$ and $\left(\sigma_{X}^{2}, \sigma_{Y}^{2}\right)$, respectively; thus, the parameter vector is $\omega=\left(\mu_{X}, \mu_{Y}, \sigma_{X}^{2}, \sigma_{Y}^{2}\right)$. The conditional distribution of $X$, given $Y$, is normal with conditional mean

$$
E(X \mid Y=y)=\mu_{X}+\rho(\omega)\left(y-\mu_{Y}\right) \frac{\sigma_{X}}{\sigma_{Y}},
$$


TABLE 2: The estimative VaR forecast and its coverage probability (in the bracket) of a random loss that depends on another random loss.

\begin{tabular}{lccr}
\hline & Normal & & $t$ \\
\hline Bivariate normal & $1.9951(0.9509)$ & Bivariate $t$ & $1.8636(0.9500)$ \\
Gaussian copula & $1.5273(0.9533)$ & $t$ copula & $2.4528(0.9543)$ \\
Clayton copula & $1.5359(0.9542)$ & Clayton copula & $2.4674(0.9549)$ \\
\hline
\end{tabular}

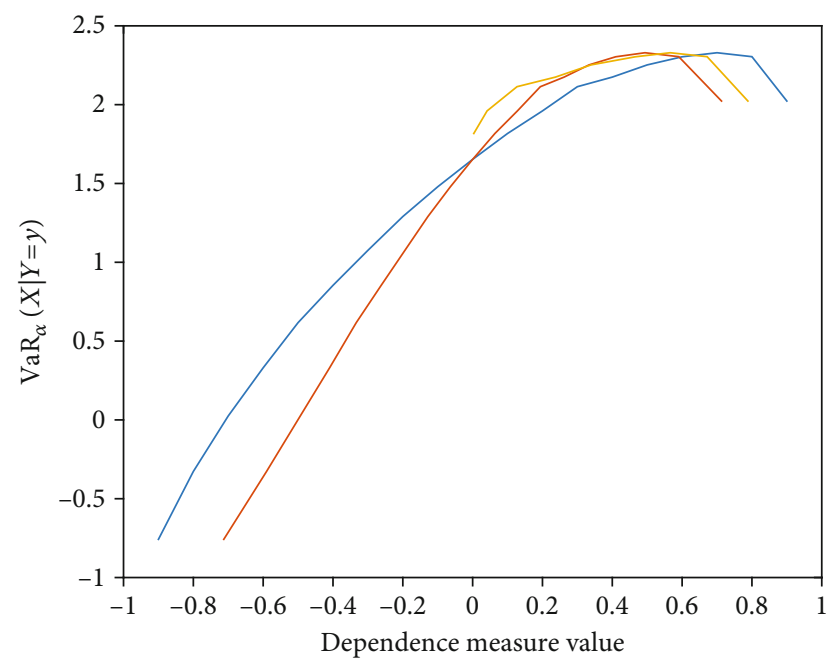

FIGURE 5: Conditional estimative VaR forecast, for bivariate normal distribution, against dependence measure $\rho(\omega)$ (blue line), compared to other dependence measures of $\tau(\omega)$ (brown line) and $\lambda_{U}(\omega)$ (yellow line); $-1 \leq \rho(\omega), \tau(\omega) \leq 1 ; 0 \leq \lambda_{U} \leq 1$.

and its corresponding conditional variance

$$
\operatorname{Var}(X \mid Y=y)=\sigma_{X}^{2}\left(1-\rho^{2}(\omega)\right)
$$

where $\rho(\omega)$ is the Pearson correlation coefficient of linear relationship between $X$ and $Y$ [16]. The estimative VaR forecast of $X \mid Y$, based on the standard deviation premium principle, at the level of significance $\alpha$ is given by

$$
\begin{aligned}
\widehat{\mathrm{VaR}}_{\alpha}(X \mid Y ; \rho(\omega))= & \widehat{\mu}_{X}+\rho(\omega)\left(y-\widehat{\mu}_{Y}\right) \frac{\widehat{\sigma}_{X}}{\widehat{\sigma}_{Y}} \\
& +\Phi^{-1}(1-\alpha) \sqrt{\widehat{\sigma}_{X}^{2}\left(1-\rho^{2}(\omega)\right)}
\end{aligned}
$$

where $\Phi^{-1}(1-\alpha)$ denotes the $(1-\alpha)$-quantile of standard normal. When $X$ and $Y$ are independent $(\rho(\omega)=0)$, we will obtain the well-known estimative $\mathrm{VaR}$ forecast for a normal random loss $X: \widehat{\mu}_{X}+\Phi^{-1}(1-\alpha) \widehat{\sigma}_{X}$, whereas when $\rho(\omega)=$ 1 (there is strong linear dependence between $X$ and $Y$ ), we have the estimative $\mathrm{VaR}$ forecast of random loss $X$, given $Y$, as just. Note that by taking the first derivative of the estimative $\mathrm{VaR}$ forecast with respect to $\rho(\omega)$, i.e.,

$$
\begin{aligned}
\frac{\partial \operatorname{VaR}_{\alpha}(X \mid Y ; \rho(\omega))}{\partial \rho(\omega)}= & \left(y-\widehat{\mu}_{Y}\right) \frac{\widehat{\sigma}_{X}}{\widehat{\sigma}_{Y}} \\
& -\Phi^{-1}(1-\alpha) \widehat{\sigma}_{X} \frac{\rho(\omega)}{\sqrt{1-\rho^{2}(\omega)}}
\end{aligned}
$$

and normalize it (equalized to zero), we obtain the value of $\rho(\omega)$ that maximizes the estimative $\mathrm{VaR}$ forecast that is

$$
\rho(\omega)_{\max }=\frac{y-\widehat{\mu}_{Y}}{\sqrt{\widehat{\sigma}_{Y}^{2}\left(\Phi^{-1}(1-\alpha)\right)^{2}+\left(y-\mu \wedge_{Y}\right)^{2}}} .
$$

It is interesting to observe the impact of dependence measure $\rho(\omega)$ to the behavior of the conditional estimative VaR forecast (see Figure 5). The stronger the dependence of $Y$ to $X$ (in the positive direction), the larger the conditional mean of $X$, given $Y$, whereas the large value of $\rho(\omega)$ may reduce the weight of conditional variance of $X$ which means the smaller conditional variance. In comparison to $\rho(\omega)$, other dependence measure of $\tau(\omega)$ is presented, and it is observed that the higher value of $\tau(\omega)$ (than $\rho(\omega)$ ) will give a similar estimative $\mathrm{VaR}$ forecast, for negative dependence, whilst positive dependence of $\tau(\omega)$ will give a higher estimative $\mathrm{VaR}$ forecast than $\rho(\omega)$.

3.2. $t$ Distribution. We consider $t$ distribution for both random losses of $X$ and $Y$. For simplicity, we take $\mu_{X}=\mu_{Y}=0$ and the degrees of freedom $\kappa_{X}=\kappa_{Y}=\kappa$.

The bivariate probability function of $(X, Y)$ is defined as

$$
\begin{aligned}
f_{X, Y}(x, y)= & \frac{1}{\sigma_{X} \sigma_{Y} \sqrt{1-\rho(\omega)^{2}}} \frac{1}{\kappa \pi} \frac{\Gamma(1+\kappa / 2)}{\Gamma(\kappa / 2)} \\
& \cdot\left(1+\frac{\Lambda}{\kappa \sigma_{X}^{2} \sigma_{Y}^{2}\left(1-\rho(\omega)^{2}\right)}\right)^{-(1+\kappa / 2)}
\end{aligned}
$$

for $-\infty<x, y<\infty$, where $\Lambda=x^{2} \sigma_{Y}^{2}+y^{2} \sigma_{X}^{2}-2 \rho(\omega) x y \sigma_{X} \sigma_{Y}$ [17]. To obtain conditional probability function $X$, given $Y$, $f_{X \mid Y}$, we need to divide the above probability function by the probability function of $Y, f_{X, Y}(x, y) / f_{Y}(y)$.

The resulting conditional probability function is

$$
\begin{aligned}
f_{X \mid Y}(x \mid y)= & \frac{1}{\sigma_{X} \sigma_{Y} \sqrt{1-\rho(\omega)^{2}}} \frac{1}{\kappa \pi} \frac{\Gamma(1+\kappa / 2)}{\Gamma(1 / 2+\kappa / 2)}\left(1+\frac{y^{2}}{\kappa}\right)^{(1 / 2+\kappa / 2)} \\
& \times\left(1+\frac{\Lambda}{\kappa \sigma_{X}^{2} \sigma_{Y}^{2}\left(1-\rho(\omega)^{2}\right)}\right)^{-(1+\kappa / 2)},-\infty<x<\infty,
\end{aligned}
$$

where its conditional mean and variance do not have explicit form and thus must be obtained numerically. In Figure 6, the effect of correlation to the estimative VaR forecast is illustrated. As $\rho(\omega)$ tends to have a positive strong correlation, 


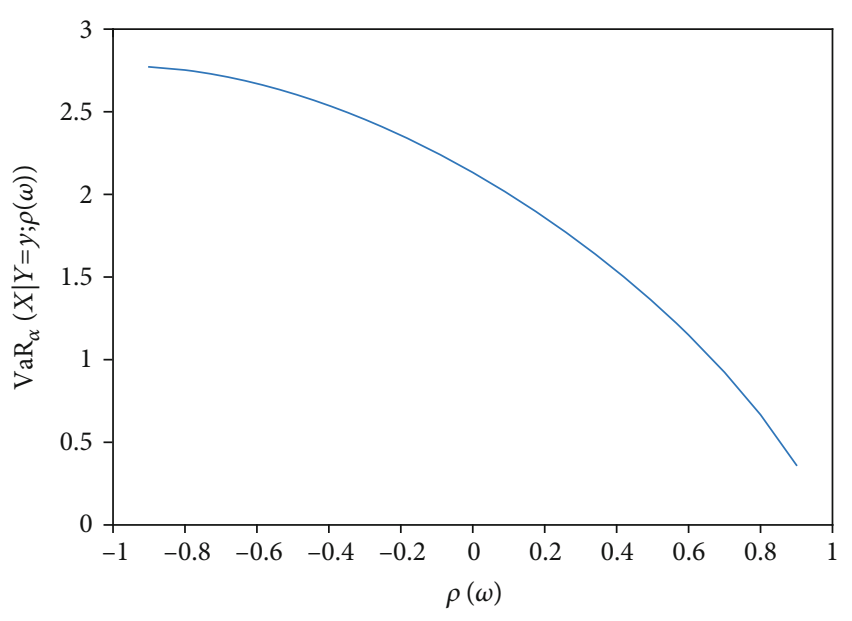

Figure 6: The estimative $\mathrm{VaR}$ forecast of $t$ distributed data against dependence measure $\rho(\omega)$; the degrees of freedom are set $\kappa_{X}=\kappa_{Y}=\kappa$.

the estimative $\mathrm{VaR}$ forecast is lower. It is typical of heavytailed $t$ distributed data, commonly in financial data, to reduce the effect on a random loss by another (dependent) random loss.

3.3. Joint Distribution of Normal and $t$. In practice, the two random losses $X$ and $Y$ may have nonidentical (marginal) distributions. For example, $X$ may be normally distributed whilst $Y$ has $t$ distribution or vice versa. Thus, the use of a copula is unavoidable to obtain the joint distribution function of such nonidentical losses, and the conditional distribution function can be derived.

Let $F_{X}$ and $G_{Y}$ be the marginal distribution function of $X$ and $Y$, respectively. As we mention in Section 2, the joint distribution function of $(X, Y)$ can be represented, through copula $C_{\tau(\omega)}$ with dependence measure $\tau(\omega)$, as

$$
H_{X, Y ; \omega}(x, y)=C_{\tau(\omega)}\left(F_{X}(x), G_{Y}(y)\right)=C_{\tau(\omega)}(u, v),
$$

where $u=F_{X}(x)$ and $v=G_{Y}(y)$. Note that the choice of $\tau(\omega)$ is because of its invariant property under nonlinear monotonic transformation; in other words, it is copula-based dependence measure [3]. From [18], by taking the first derivative of $C_{\tau(\omega)}(u, v)$ with respect to $v$, we can obtain the conditional distribution function of $X$, given $Y$, i.e.,

$$
\begin{aligned}
F_{X \mid Y ; \omega}(x \mid y) & =\frac{\partial C_{\tau(\omega)}(u, v)}{\partial v}=\frac{\partial C_{\tau(\omega)}\left(F_{X}(x), G_{Y}(y)\right)}{\partial G_{Y}(y)} \\
& =C_{X \mid Y ; \tau(\omega)}\left(F_{X}(x) \mid G_{Y}(y)\right) .
\end{aligned}
$$

Here, the function $C_{X \mid Y ; \tau(\omega)}$ is the conditional distribution function of $U=F_{X}(X)$, given $V=G_{Y}(Y)$. For Archimedean, Gaussian, and $t$ copulas, based on [19], each function $C_{X \mid Y ; \tau(\omega)}$ is given in Table 3.
Based on the conditional distribution function defined above, the conditional $\mathrm{VaR}$ of $X$, given $Y$, at a level of significance $\alpha$ can be derived from

$$
\begin{aligned}
1-\alpha & =F_{X \mid Y ; \omega}(\operatorname{VaR}(X \mid Y=y ; \tau(\omega)) \mid y) \\
& =C_{X \mid Y ; \tau(\omega)}\left(F_{X}\left(\operatorname{VaR}(X \mid Y=y ; \tau(\omega)) \mid G_{Y}(y)\right)\right),
\end{aligned}
$$

and we obtain

$$
\operatorname{VaR}(X \mid Y=y ; \tau(\omega))=F_{X}^{-1}\left(C_{X \mid Y ; \tau(\omega)}^{-1}\left(1-\alpha \mid G_{Y}(y)\right)\right)
$$

Because the parameter of the copula can be expressed as the function of dependence measure $\tau(\omega)$ (see the third column of Table 3), it is clear that $F_{X}^{-1}\left(C_{X \mid Y ; \tau(\omega)}^{-1}\left(1-\alpha \mid G_{Y}(y)\right)\right)$ in (16) consists $\tau(\omega)$ and so the conditional VaR of $X \mid Y$.

If $X$ is normally distributed whilst $Y$ has $t$ distribution, we get the conditional estimative $\mathrm{VaR}$ forecast:

$$
\begin{aligned}
& \widehat{\mathrm{VaR}}(X \mid Y=y ; \tau(\omega)) \\
& \quad=\widehat{\mu}_{X}+\widehat{\sigma}_{X} \Phi^{-1}\left(C_{X \mid Y ; \tau(\omega)}^{-1}\left(1-\alpha \mid t_{\kappa}\left(\frac{y-\widehat{\mu}_{Y}}{\widehat{\sigma}_{Y}}\right)\right)\right) .
\end{aligned}
$$

If $X$ has $t$ distribution whilst $Y$ is normally distributed, the conditional estimative $\mathrm{VaR}$ forecast can be expressed as

$$
\begin{aligned}
& \widehat{\operatorname{VaR}}(X \mid Y=y ; \tau(\omega)) \\
& \quad=\widehat{\mu}_{X}+\widehat{\sigma}_{X} t_{\kappa}^{-1}\left(C_{X \mid Y ; \tau(\omega)}^{-1}\left(1-\alpha \mid \Phi\left(\frac{y-\widehat{\mu}_{Y}}{\widehat{\sigma}_{Y}}\right)\right)\right) .
\end{aligned}
$$

For both cases, the effect of dependence to the conditional estimative $\mathrm{VaR}$ forecast is illustrated in Figure 7, by using Gumbel, Clayton, Gaussian, and $t$ copulas. The stronger the dependence of random loss to others (in negative or positive sign), the lower the conditional estimative VaR forecast. It happens when Clayton, Gaussian, and $t$ copulas are used. If we use the Clayton copula, the conditional estimative $\mathrm{VaR}$ forecast always decreases as the value of $\tau(\omega)$ increases.

\section{The Case of Risk Model with Volatility: ARCH}

In statistical modeling, random losses may be represented as a stochastic process $\left\{X_{t}\right\}$ denoted as asset returns: $X_{t}=\sigma_{t} \varepsilon_{t}$, where $\varepsilon_{t}$ is white noise with zero mean and unit variance, e.g., [1]. Suppose that the available data are $X_{1}, X_{2}, \cdots, X_{n}$. Thus, the estimative $\mathrm{VaR}$ forecast for $X_{n+1}$, given observations up to time $n, \mathfrak{F}_{n}$, is

$$
\widehat{\operatorname{VaR}}_{\alpha}\left(X_{n+1} \mid \mathfrak{\mho}_{n} ; \rho(\omega)\right)=\widehat{\sigma}_{n+1} \cdot F_{\varepsilon}^{-1}(1-\alpha),
$$

where $\widehat{\sigma}_{n+1}$ denotes the one-step-ahead volatility forecast; $\rho(\omega)$ is the dependence measure between $X_{i}$ and $X_{j}$ (with $\omega$ as the parameter vector of an asset return model), for $i \neq$ $j$; and $F_{\varepsilon}^{-1}(1-\alpha)$ is $(1-\alpha)$-quantile of distribution of $\varepsilon_{t}$. 
TABLE 3: Conditional function $\left.C_{X}\right|_{Y ; \tau(\omega)}$ for Archimedean, Gaussian, and $t$ copulas and the expression of its parameter as the function of $\tau(\omega)$.

\begin{tabular}{|c|c|c|}
\hline & Conditional function $C_{X \mid Y ; \tau(\omega)}(u \mid v)$ & Parameter \\
\hline G & $v^{-1}(-\ln (v))^{\theta-1}\left((-\ln (u))^{\theta}+(-\ln (v))^{\theta}\right)^{(1 / \theta)-1} C_{\tau(\omega)}^{\mathrm{G}}(u, v)$ & $\theta=\frac{1}{1-\tau(\omega)}$ \\
\hline $\mathrm{C}$ & $v^{-\theta-1}\left(u^{-\theta}+v^{-\theta}-1\right)^{-(1 / \theta)-1}$ & $\theta=\frac{2 \tau(\omega)}{1-\tau(\omega)}$ \\
\hline $\mathrm{Ga}$ & $\Phi\left(\frac{\Phi^{-1}(u)-\theta \Phi^{-1}(v)}{\sqrt{1-\rho(\omega)^{2}}}\right)$ & $\rho(\omega)=\sin \left(\frac{\pi}{2} \tau(\omega)\right)$ \\
\hline$t$ & $t_{\kappa+1}\left(\frac{t_{\kappa}^{-1}(u)-\theta t_{\kappa}^{-1}(v)}{\sqrt{\left(1-\rho(\omega)^{2}\right)\left(\kappa+\left(t_{\kappa}^{-1}(v)\right)^{2}\right) /(\kappa+1)}}\right)$ & $\rho(\omega)=\sin \left(\frac{\pi}{2} \tau(\omega)\right)$ \\
\hline
\end{tabular}

Unlike in Section 3, now the dependence is between $X=X_{n+1}$ and $Y=X_{n}$ in which the literatures show that no or little dependence occurs. Note that the VaR forecast formula (19) does not include conditional mean $E\left(X_{n+1} \mid \mathrm{F}_{n}\right)$ since this has zero value. As for the volatility process, we consider an $\mathrm{ARCH}(1)$ model; its forecast is given by

$$
\widehat{\sigma}_{n+1}=\sqrt{\widehat{a}_{0}+\widehat{a}_{1} X_{n}^{2}}
$$

which can be observed as conditional only on the last observation, $X_{n}$, since the Markov property applies to the ARCH model. Table 4 and its corresponding Figure 8 show the conditional coverage probability of the estimative $\mathrm{VaR}$ forecast.

4.1. Two Dependent Stochastic Processes. We may also consider the case of stochastic processes $\{X t\}$ and $\{Y t\}$ which are dependent with certain dependence measures such as Kendall's $\tau(\omega)$, where $\omega$ is the parameter vector of the model. In other words, we aim to find the estimative VaR forecast of $X_{n+1}$, given $Y_{n+1}$ :

$$
\begin{aligned}
& \operatorname{VaR}_{\alpha}\left(X_{n+1} \mid Y_{n+1} ; \tau(\omega)\right) \\
& \quad=E\left(X_{n+1} \mid Y_{n+1}\right)+\Phi^{-1}(1-\alpha) \sqrt{\operatorname{VaR}\left(X_{n+1} \mid Y_{n+1}\right)}
\end{aligned}
$$

with nonzero conditional mean and time-varying conditional variance. For simplicity, we take an $\mathrm{ARCH}(1)$ model for both processes. Provided an ARCH(1)-normal model, $X_{n+1}$ is normally distributed with mean $\mu_{X_{n+1} \mid X_{n}}$ and variance $\sigma_{X_{n+1} \mid X_{n}}^{2}$

Now, the conditional mean of $X_{n+1}$, given $Y_{n+1}$, with dependence measure $\tau(\omega)$ is given as

$$
E\left(X_{n+1} \mid Y_{n+1}=y\right)=\mu_{X_{n+1} \mid X_{n}}+\tau(\omega)\left(y-\mu_{Y_{n+1} \mid Y_{n}}\right) \frac{\sigma_{X_{n+1} \mid X_{n}}}{\sigma_{Y_{n+1} \mid Y_{n}}}
$$

where $\tau(\omega)=(2 / \pi) \arcsin (\rho(\omega))$ (see [20]); its corresponding conditional variance is

$\operatorname{VaR}\left(X_{n+1} \mid Y_{n+1}=y\right)=\sigma_{X_{n+1} \mid X_{n}}^{2}\left(1-\left(\frac{2}{\pi}\right)\right) \arcsin \left(\rho^{2}(\omega)\right)$

Note that the choice of $\tau$ instead of $\rho$ is mainly due to the possibility of nonlinear dependence among two processes. In Figure 9, we observe the coverage probability of the estimative $\mathrm{VaR}$ forecast of two ARCH(1) processes. The simulation result shows quite close conditional coverage properties (red line) to the true coverage.

\section{Estimative VaR Forecast of Two Losses with Tail Dependence}

We have so far consider the calculation of the estimative VaR forecast as an upper prediction limit with a certain level of significance; the parameter of distribution or model is typically estimated by the maximum likelihood (ML) method. The accuracy of such VaR forecast may be computed through coverage probability that takes parameter variability into account (and the ML method is the common approach). Some authors, e.g., $[5-7,21]$, have done the effect of parameter variability into coverage probability accuracy for independent observations and heteroscedastic processes. We propose here to observe the effect of dependence measure for the case of two random losses, in particular when they have tail dependence.

To gain more tail dependence effect on $\mathrm{VaR}$ forecast computation, we first briefly show the linear correlation behavior for whole data. We have usually assumed that a single correlation coefficient is appropriate to cover dependence behavior for such data. Figure 10 explains several types of data with a single correlation line (that means single correlation coefficient, $\rho=0.9,0.5$, and -0.8$)$. We, of course, hope that such correlation line will represent whole data, i.e., each observation is close to the line. However, there is possibility that a single correlation line or coefficient may not be appropriate (see Figure 10(b)). 


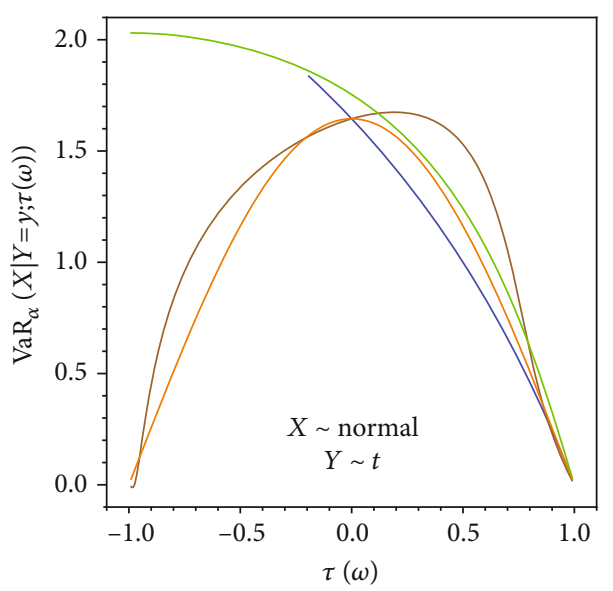

(a)

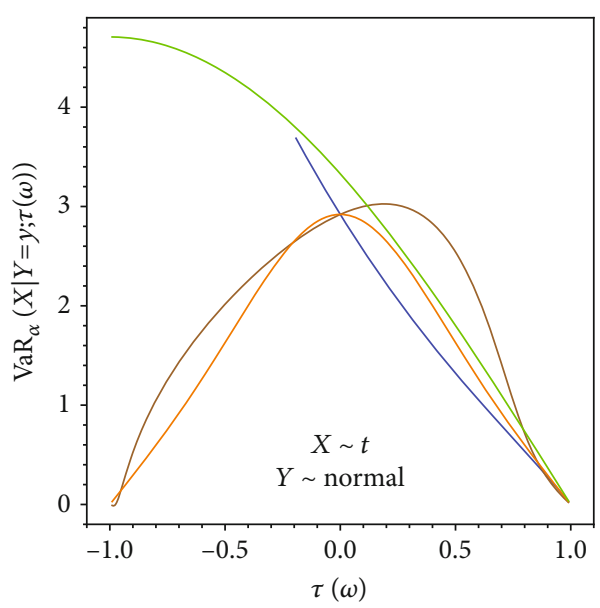

(b)

Figure 7: Conditional estimative VaR forecast, for $X \sim$ normal and $Y \sim t$ (a), also for $X \sim t$ and $Y \sim$ normal (b), against dependence measure $\tau(\omega)$ by using Gumbel (blue line), Clayton (brown line), Gaussian (yellow line), and $t$ copulas.

TABLE 4: The coverage probability of estimative VaR forecast of $\mathrm{ARCH}(1)$ process for several $(1-\alpha)$, ranging from 0.90 to 0.99 .

\begin{tabular}{lcccc}
\hline 0.90 & 0.93 & 0.95 & 0.97 & 0.99 \\
\hline 0.89609 & 0.92654 & 0.947024 & 0.967739 & 0.988887 \\
0.900009 & 0.930008 & 0.950007 & 0.970005 & 0.990002 \\
0.891878 & 0.922777 & 0.943759 & 0.965222 & 0.987607 \\
0.901274 & 0.93112 & 0.950958 & 0.970721 & 0.990347 \\
0.899503 & 0.929562 & 0.949625 & 0.969717 & 0.989863 \\
0.901369 & 0.931441 & 0.951231 & 0.970926 & 0.990446 \\
0.898208 & 0.928418 & 0.948643 & 0.968973 & 0.989499 \\
0.896911 & 0.927268 & 0.947653 & 0.968219 & 0.989126 \\
0.901066 & 0.930938 & 0.950802 & 0.970604 & 0.990291 \\
\hline
\end{tabular}

Now, we observe another direction of dependence behavior for the data and thus the effect on calculating the estimative VaR forecast. Specifically, we rely on tail distribution for the dependence among losses; single dependence value but only for some part of data. The proposed idea is motivated by the fact that two random losses may have dependence only in part of the data, say in the tail of its distribution. To illustrate, we describe in Figure 11 in which two losses have used different dependence measures. As before, the key point here is the joint distribution in which we most likely have to rely on a copula instead of the classic joint distribution function; the Clayton copula may capture the lower tail dependence whilst the Gumbel copula shows the distribution to have upper tail dependence.

Suppose that two random losses, $X$ and $Y$, are dependent with parameter vector $\omega$. McNeil et al. [3] and Lai et al. [15], for instance, have a formal definition of upper TDC between $X$ and $Y$ as

$$
\lambda_{U}(X, Y)=\lambda_{U}=\lim _{\alpha \rightarrow 0^{+}} P\left(X>F_{X}^{-1}(1-\alpha) \mid Y>G_{Y}^{-1}(1-\alpha)\right)
$$

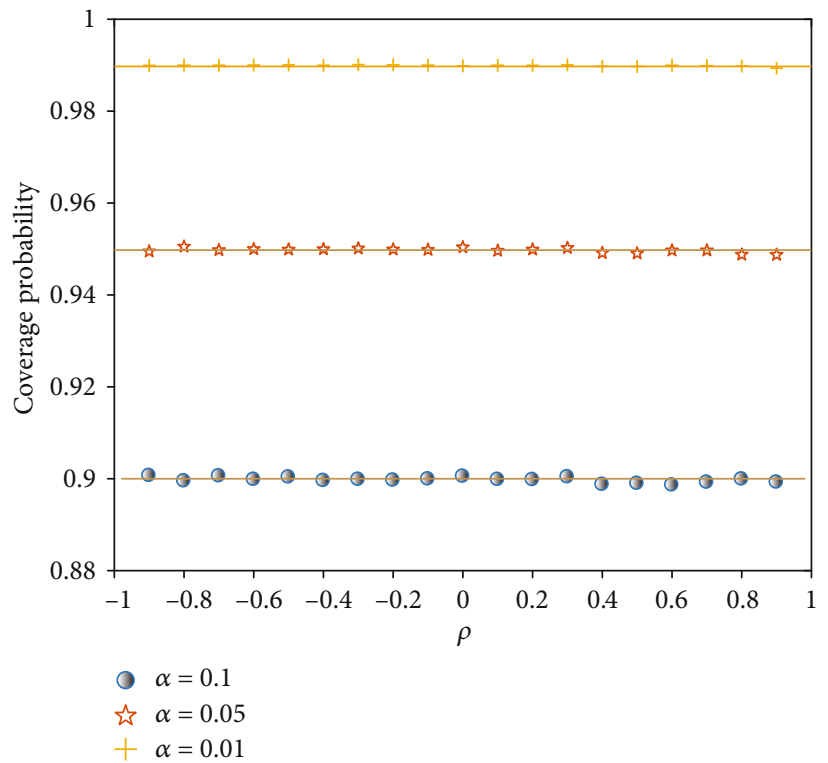

FIgURE 8: The coverage probability of estimative VaR forecast for the case of the $\mathrm{ARCH}(1)$ process; $1-\alpha=0.9$ (blue), 0.95 (brown), and 0.99 (yellow).

provided that the limit exists. The tail coefficient is ranging from zero to one, i.e., $0 \leq \lambda_{U} \leq 1$. Note that $F^{-1}$ and $G^{-1}$ are the inverse of distribution function for $X$ and $Y$, respectively. When the coefficient is zero, i.e., $\lambda_{U}=0$, then we would say that two losses are asymptotically independent.

The upper TDC formula involving the Gumbel copula, for example, will be defined as

$$
\lambda_{U}(\omega)=\lim _{\alpha \rightarrow 0^{+}} \frac{\bar{C}_{\omega}^{\mathrm{G}}(\alpha, \alpha)}{\alpha}=2-2^{1 / \omega}
$$

where $\bar{C}$ denotes the surviving copula and $\omega$ is the parameter of the Gumbel copula. Figure 11 describes Gumbel distributed data against linear correlation coefficient and tail 


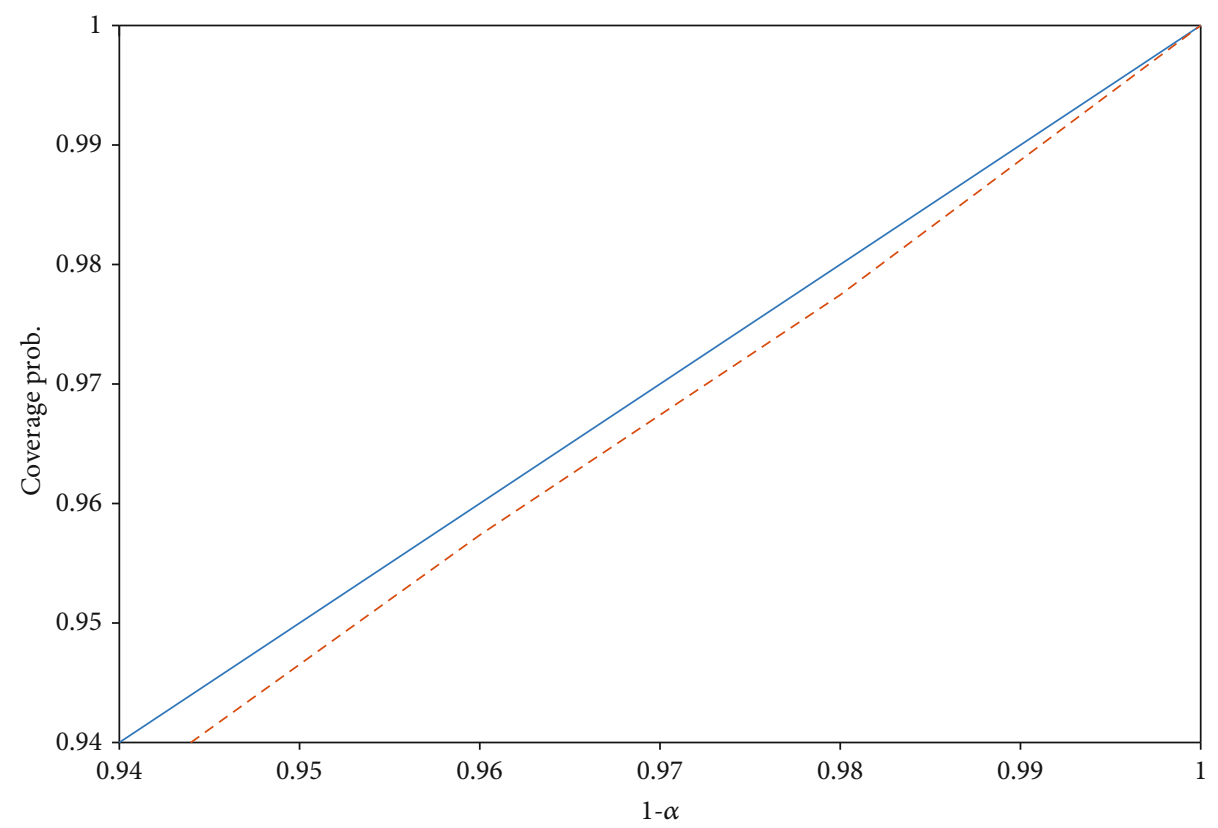

Figure 9: The conditional coverage probability of the estimative VaR forecast developed from two ARCH(1) processes; the estimated coverage (dashed red line) is quite close to the true coverage (solid blue line).

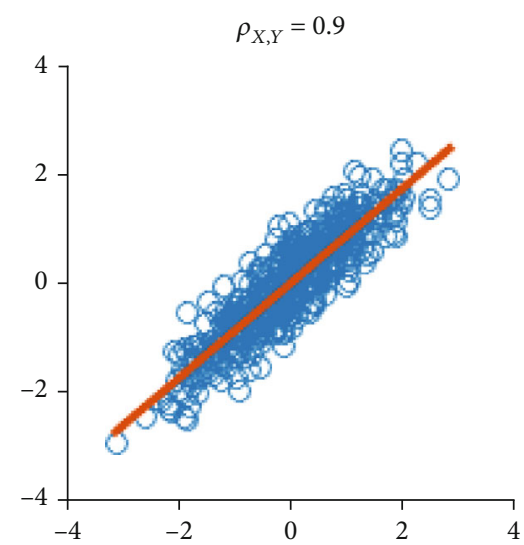

(a)

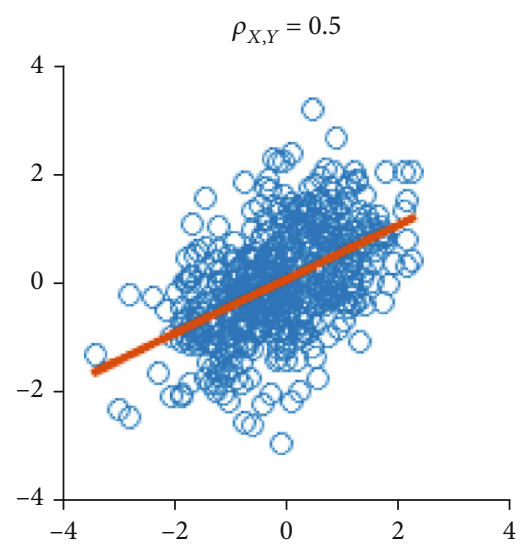

(b)

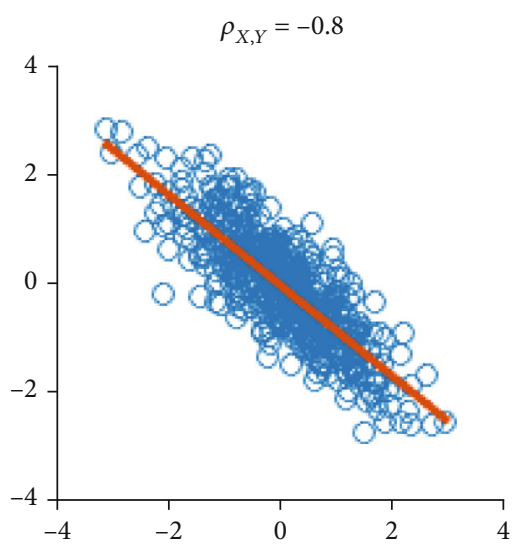

(c)

Figure 10: Single correlation line or coefficient for several types of data; $\rho=0.9,0.5$, and -0.8 .

dependence. We show the behavior of linear correlation in "adapting" (to adjust) data behavior. In Figure 11(a), there is only one correlation coefficient for each figure $(\rho \omega=$ 0.5767 , top; $\rho \omega=0.8866$, bottom), whereas there are two correlation coefficients for each figure in Figure 11(b) to adjust data generated from the Gumbel copula. It may be explained as follows (for example, Figure 11(b), bottom figure): data generated with upper tail dependence coefficient $\lambda_{U}(\omega)=$ 0.8 may have similar behavior with composite data with a lower correlation coefficient $\rho_{L}=0.8281$ and upper correlation coefficient $\rho_{U}=0.6104$; in other words, these lower and upper correlation coefficients may be more appropriate than a single correlation coefficient.

The estimative $\mathrm{VaR}$ forecasts of $X$, given $Y$, along with correlation, rank, and upper tail dependence coefficient are shown in Table 5; its backtesting (Table 6) is carried out via Correct-VaR (e.g., see [22]). As the correlation coefficient (and the rank and upper tail dependence coefficients) is higher, so is the estimative $\mathrm{VaR}$ forecast for a single random loss. The accuracy, however, may not be accurate. In comparison, the Correct-VaR for dependent random loss is quite accurate and close to $\alpha=0.05$. The stronger the dependence of random loss to others (in negative or positive signs), the better the coverage properties of their estimative $\mathrm{VaR}$ forecasts.

\section{Illustration of VaR Forecast for Real Data}

We carry out numerical study on a data set that contains negative daily log-returns of Standard \& Poor's 500 (or S\&P 500) and Dow Jones stock market indexes for the time period 2015-2019. The data set is obtained from Yahoo Finance. 

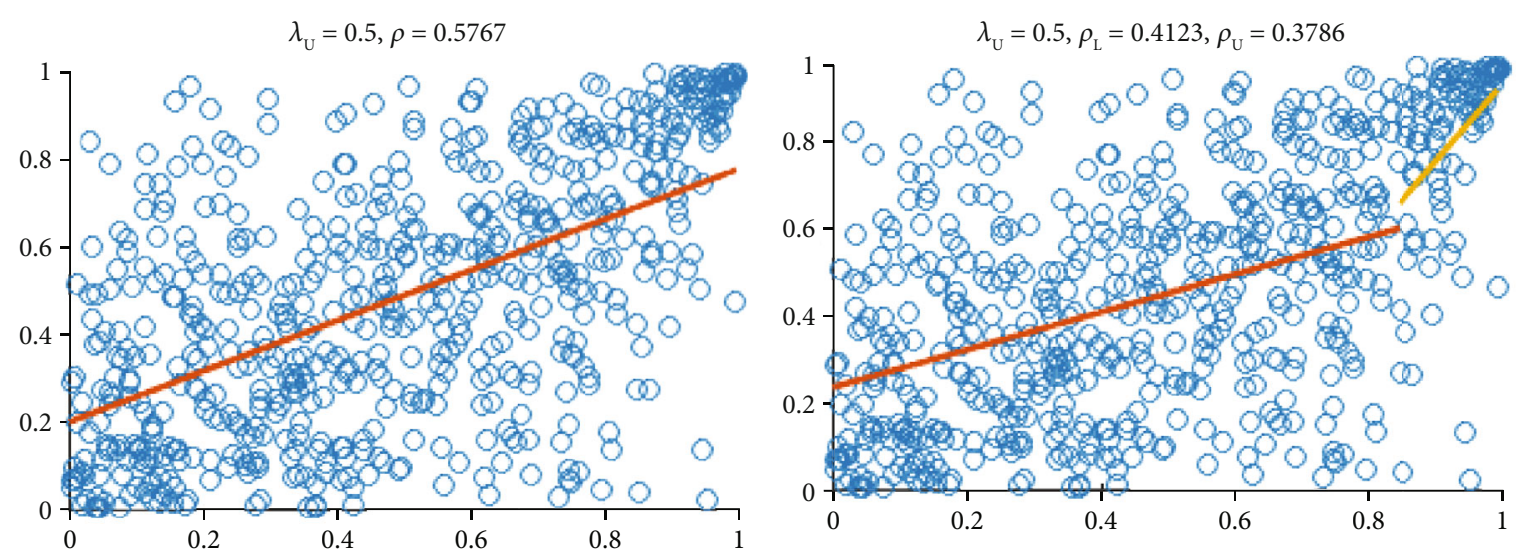

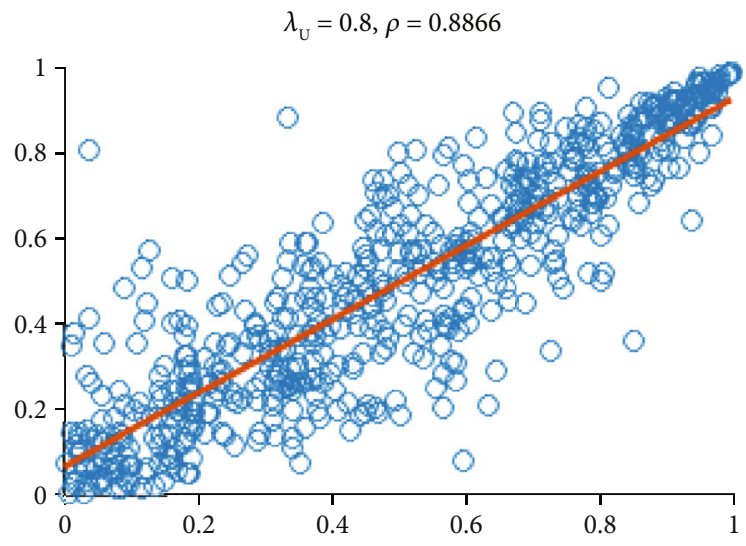

(a)

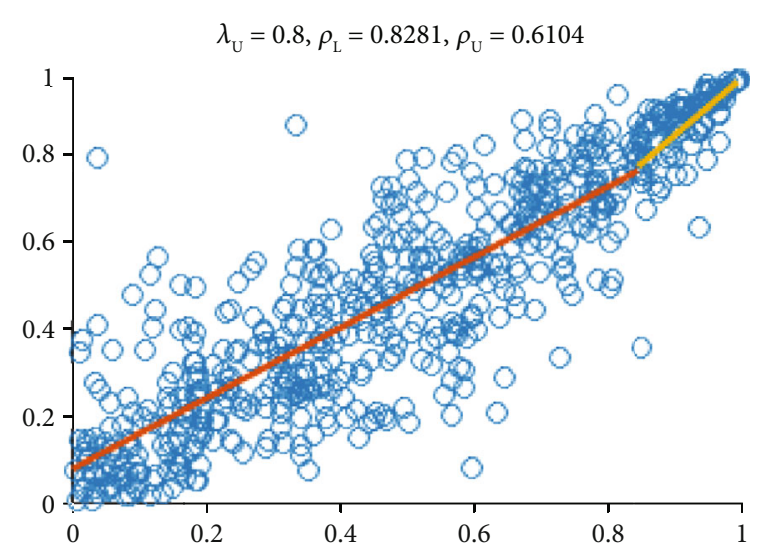

(b)

FIgURE 11: Correlation line(s) for the Gumbel copula generated data with upper tail dependence coefficient; (b) shows two correlation coefficients, upper and lower, to cover dependence behavior of the whole data.

TABle 5: Correlation, rank, and upper tail dependence-based estimative $\mathrm{VaR}$ forecast for a single random loss and a random loss that depends on another random loss.

\begin{tabular}{lcccc}
\hline$\rho(\omega)$ & $\tau(\omega)$ & $\lambda_{U}(\omega)$ & $\widehat{\mathrm{VaR}}_{\alpha}(X ; \theta)$ & $\widehat{\mathrm{VaR}}_{\alpha}(X \mid Y=y ; \rho(\omega))$ \\
\hline-0.9 & -0.7129 & 0 & 1.6450 & -0.7577 \\
-0.6 & -0.4097 & 0 & 1.6656 & 0.3306 \\
-0.5 & -0.3333 & 0 & 1.6450 & 0.6172 \\
-0.1 & -0.0638 & 0 & 1.6478 & 1.4791 \\
0 & 0 & 0 & 1.6499 & 1.6528 \\
0.2 & 0.1282 & 0.0413 & 1.6452 & 1.9603 \\
0.3 & 0.1940 & 0.1271 & 1.6494 & 2.1140 \\
0.5 & 0.3333 & 0.3391 & 1.6503 & 2.2522 \\
0.8 & 0.5903 & 0.6716 & 1.6585 & 2.3044 \\
0.9 & 0.7129 & 0.7884 & 1.6582 & 2.0230 \\
\hline
\end{tabular}

First, we assume that the negative daily log-returns are i.i.d. observations. The goodness-of-fit of the data can be determined visually in Figure 12. We can see that each data set fits to $t$ distribution.

Since the marginal distributions are from the same family $(t)$, we consider bivariate $t$ for their joint distribution. As comparison, the scatter plot in Figure 13 shows that the $t$ copula can also be used because the transformed data have both upper and lower tail dependence. The (conditional) estimative $\mathrm{VaR}$ forecasts of the negative daily log-return of the S\&P 500 index, given the negative daily log-return of the Dow Jones index, are summarized in Table 7. As we can see, for each level of significance, the use of the $t$ copula implies the larger conditional estimative $\mathrm{VaR}$ forecast than the forecast through the bivariate $t$ distribution.

Now, Figure 14 shows that the negative daily log-return data have volatility clustering and time-varying properties. Hence, we also assume that the data follow a heteroscedastic process; here, the $\mathrm{ARCH}(1)$ process is considered. The (conditional) estimative $\mathrm{VaR}$ forecasts are summarized in Table 8.

Note that all the (conditional) estimative VaR forecast above have been evaluated through its conditional coverage probability, as shown in the bracket, or Correct-VaR. Such forecast accuracy is computed by the method as in $[5,6$, 23]. The best or more appropriate $\mathrm{VaR}$ forecast is determined by the closeness of coverage probability with the target value of $1-\alpha$. The use of the copula, in Table 7, suggests larger VaR forecast with the consequence of less accuracy.

\section{Discussion}

The estimative VaR may be calculated based on the quantile of its distribution. In addition, the effect of dependence 
TABLE 6: The accuracy of estimative VaR forecasts via Correct-VaR; its value corresponds to $\alpha$ as this refers to the number of violations, i.e., the number of observations above the estimative VaR forecast.

\begin{tabular}{lcccc}
\hline$\rho(\omega)$ & $\tau(\omega)$ & $\lambda_{U}(\omega)$ & $\operatorname{Correct}_{-V_{\alpha}}(X ; \widehat{\theta})$ & Correct-VaR $_{\alpha}^{c}(X \mid Y=y ; \rho(\omega))$ \\
\hline-0.9 & -0.7129 & 0 & 0.0580 & 0.0510 \\
-0.6 & -0.4097 & 0 & 0.0430 & 0.0550 \\
-0.5 & -0.3333 & 0 & 0.0530 & 0.0540 \\
-0.1 & -0.0638 & 0 & 0.0410 & 0.0470 \\
0 & 0 & 0 & 0.0420 & 0.0470 \\
0.2 & 0.1282 & 0.0413 & 0.0540 & 0.0490 \\
0.3 & 0.1940 & 0.1271 & 0.0380 & 0.0490 \\
0.5 & 0.3333 & 0.3391 & 0.0550 & 0.0450 \\
0.8 & 0.5903 & 0.6716 & 0.0550 & 0.0530 \\
0.9 & 0.7129 & 0.7884 & 0.0390 & 0.0490 \\
\hline
\end{tabular}

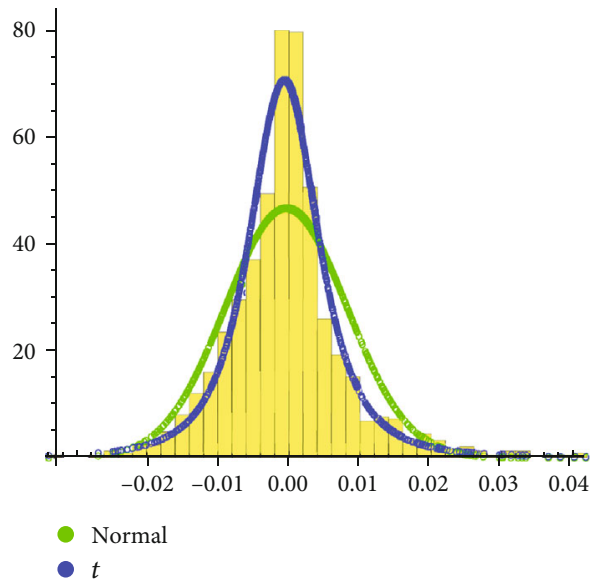

(a)

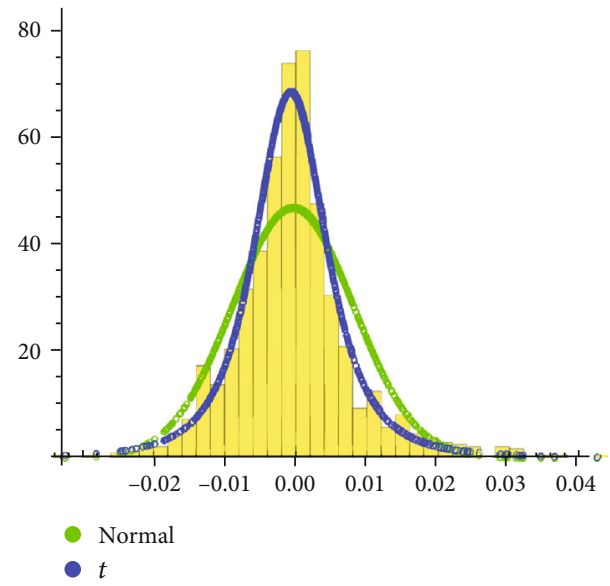

(b)

Figure 12: Histogram of the negative daily log-returns of the S\&P 500 (a) and Dow Jones (b); each data set fits to the $t$ distribution (represented by the blue probability function) better than the normal distribution (represented by the green probability function).

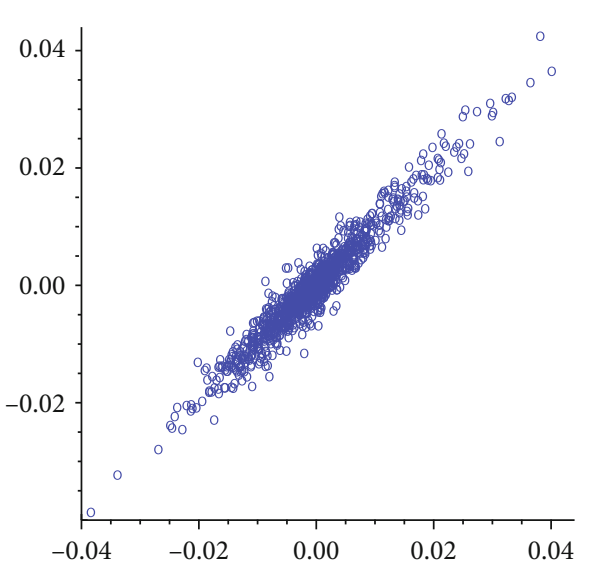

(a)

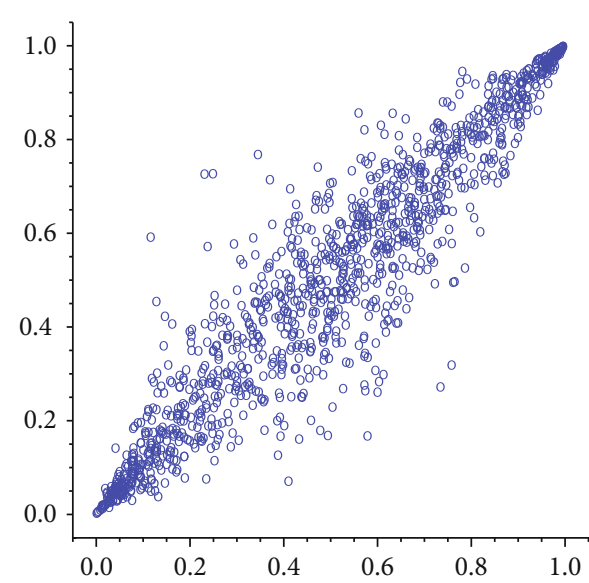

(b)

Figure 13: The scatter plot of the negative daily log-returns of the S\&P 500 index versus the Dow Jones index (a) and their transformations into uniformly distributed data (b) that have both upper and lower tail dependence. 
TABLE 7: The (conditional) estimative VaR forecast and its coverage probability (in the bracket) of the negative daily log-returns of the S\&P 500 index, given the negative daily log-returns of the Dow Jones index, with i.i.d. assumption; the conditional estimative VaR forecast in the last column is calculated through $t$ copula.

\begin{tabular}{llcc}
\hline $1-\alpha$ & $\widehat{\mathrm{VaR}}_{\alpha}(X ; \rho(\omega))$ & $\widehat{\mathrm{VaR}}_{\alpha}(X \mid Y=y ; \rho(\omega))$ & $\widehat{\mathrm{VaR}}_{\alpha}^{c}(X \mid Y=y ; \rho(\omega))$ \\
\hline 0.90 & $0.0081(0.9019)$ & $0.0022(0.8989)$ & $0.0029(0.7959)$ \\
0.93 & $0.0101(0.9308)$ & $0.0026(0.9290)$ & $0.0035(0.8624)$ \\
0.95 & $0.0121(0.9499)$ & $0.0030(0.9504)$ & $0.0041(0.9022)$ \\
0.97 & $0.0156(0.9687)$ & $0.0036(0.9708)$ & $0.0050(0.9400)$ \\
0.99 & $0.0254(0.9896)$ & $0.0050(0.9915)$ & $0.0073(0.9789)$ \\
\hline
\end{tabular}

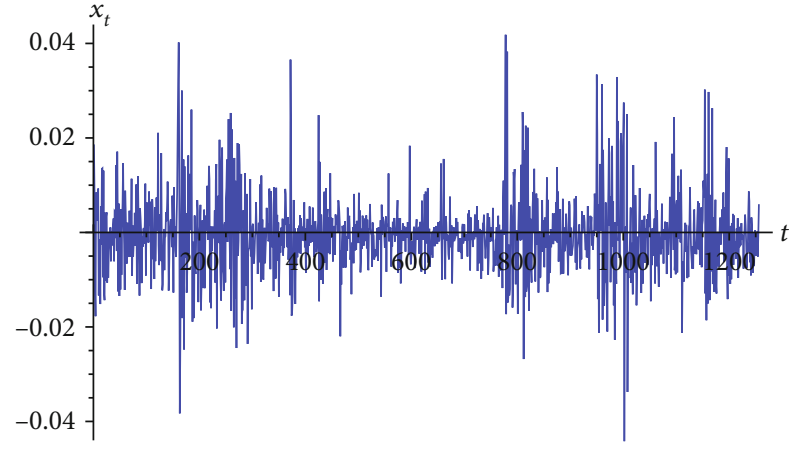

(a)

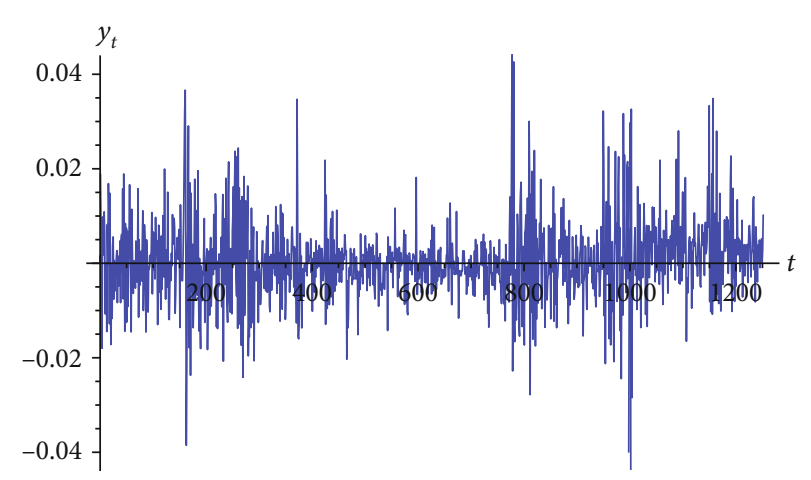

(b)

FIgURE 14: The time series plot of the negative daily log-returns of the S\&P 500 (a) and Dow Jones (b) indexes.

TABLE 8: The (conditional) estimative VaR forecast and its coverage probability (in the bracket) of the negative daily log-returns of the S\&P 500 index, given the negative daily log-returns of the Dow Jones index, by assuming $\mathrm{ARCH}(1)$ processes.

\begin{tabular}{lcc}
\hline $1-\alpha$ & $\widehat{\mathrm{VaR}}_{\alpha}\left(X_{n+1} \mid \mathfrak{F}_{n} ; \rho(\omega)\right)$ & $\widehat{\mathrm{VaR}}_{\alpha}\left(X_{n+1} \mid Y_{n+1} ; \rho(\omega)\right)$ \\
\hline 0.90 & $0.0099(0.8976)$ & $0.0025(0.8977)$ \\
0.93 & $0.0114(0.9303)$ & $0.0029(0.9288)$ \\
0.95 & $0.0127(0.9512)$ & $0.0032(0.9482)$ \\
0.97 & $0.0150(0.9683)$ & $0.0037(0.9682)$ \\
0.99 & $0.0179(0.9896)$ & $0.0045(0.9899)$ \\
\hline
\end{tabular}

(such as Pearson's correlation, Kendall's tau, or tail dependence) occurs for the VaR forecast of two dependent random losses or stochastic process. Furthermore, the use of a copula is significant due to the fact that the classical joint distribution function may not be explicitly formulated. However, it has consequence of reducing forecast accuracy. As for the efficiency of the $\mathrm{VaR}$ forecast, it may be applied when we consider several models such as ARCH/GARCH with different assumptions of innovation: normal $(\mathrm{N})$, skewed normal $(\mathrm{SN})$, skewed $t(\mathrm{ST})$, generalized error distribution (GED), etc.

Kuan et al. [24] pointed out the expectile-based VaR forecast, say eVaR. This considers the magnitude of losses instead of just the probability of losses. There is a possibility that the measure of dependence is incorporated to the eVaR forecast. It is of course because the tail dependence and corresponding copula play an important role.

\section{Data Availability}

S\&P 500 index and Dow Jones index data have been used.

\section{Conflicts of Interest}

The authors declare that they have no conflicts of interest.

\section{Acknowledgments}

We are grateful for financial support of "HIbah Berbasis Kompetensi" from the Ministry of Research, Technology and Higher Education of the Republic of Indonesia, 20172018. We are indebted to Arief Hakim for providing joint distribution of normal and $t$ and $\mathrm{VaR}$ forecast calculation of real data.

\section{References}

[1] P. Christoffersen and S. Gonçalves, Estimation Risk in Financial Risk Management, CIRANO Scientific Series, 2004.

[2] J.-J. Huang, K.-J. Lee, H. Liang, and W.-F. Lin, "Estimating value at risk of portfolio by conditional copula-GARCH method," Insurance: Mathematics and Economics, vol. 45, no. 3, pp. 315-324, 2009.

[3] A. J. McNeil, R. Frey, and P. Embrechts, Quantitaive Risk Management: Concepts, Techniques and Tools, Princeton University Press, 2005.

[4] M. R. Nieto and E. Ruiz, "Frontiers in VaR forecasting and backtesting," International Journal of Forecasting, vol. 32, no. 2, pp. 475-501, 2016. 
[5] P. Kabaila and K. Syuhada, "Improved prediction limits for $\operatorname{AR}(p)$ and $\operatorname{ARCH}(p)$ processes," Journal of Time Series Analysis, vol. 29, no. 2, pp. 213-223, 2008.

[6] P. Kabaila and K. Syuhada, "The asymptotic efficiency of improved prediction intervals," Statistics \& Probability Letters, vol. 80, no. 17-18, pp. 1348-1353, 2010.

[7] P. Vidoni, "Improved prediction intervals for stochastic process models," Journal of Time Series Analysis, vol. 25, no. 1, pp. 137-154, 2004.

[8] W.-H. Cheng and J.-C. Hung, "Skewness and leptokurtosis in GARCH-typed VaR estimation of petroleum and metal asset returns," Journal of Empirical Finance, vol. 18, no. 1, pp. 160-173, 2011.

[9] S. H. Kang and S.-M. Yoon, "Value-at-risk analysis for Asian emerging markets: asymmetric and fat tails in return innovation," The Korean Economic Review, vol. 25, no. 2, pp. 387411, 2009.

[10] P. A. Santos and M. I. F. Fraga Alves, "Forecasting value-atrisk with a duration-based POT method," Mathematics and Computers in Simulation, vol. 94, pp. 295-309, 2013.

[11] Y.-H. H. Ku and J. J. Wang, "Estimating portfolio value-at-risk via dynamic conditional correlation MGARCH model an empirical study on foreign exchange rates," Applied Economics Letters, vol. 15, no. 7, pp. 533-538, 2008.

[12] S. B. Messaoud and C. Aloui, "Measuring risk of portfolio: GARCH-copula model," Journal of Economic Integration, vol. 30, no. 1, pp. 172-205, 2015.

[13] P. Abad and S. Benito, "A detailed comparison of value at risk estimates," Mathematics and Computers in Simulation, vol. 94, pp. 258-276, 2013.

[14] P. Jorion, "Risk2: measuring the risk in value at risk," Financial Analysts Journal, vol. 52, no. 6, pp. 47-56, 1996.

[15] Y. Lai, C. W. S. Chen, and R. Gerlach, "Optimal dynamic hedging via copula-threshold-GARCH models," Mathematics and Computers in Simulation, vol. 79, no. 8, pp. 2609-2624, 2009.

[16] J. Rice, Mathematical Statistics and Data Analysis, Duxbury, 1995.

[17] N. Balakrishnan and C. D. Lai, Continuous Bivariate Distributions, Springer, 2nd edition, 2009.

[18] C. Jiang and W. Long, "Does tail dependence make a difference in the estimation of systemic risk? $\triangle \mathrm{CoVaR}$ and MES," SSRN Electronic Journal, pp. 1-45, 2018.

[19] H. Joe, Dependence Modeling with Copulas, CRC Press, 2015.

[20] H.-B. Fang, K.-T. Fang, and S. Kotz, "The meta-elliptical distributions with given marginals," Journal of Multivariate Analysis, vol. 82, no. 1, pp. 1-16, 2002.

[21] K. Syuhada and R. Saputra, "On prediction interval for independent observations," Applied Mathematical Sciences, vol. 9, no. 99, pp. 4931-4940, 2015.

[22] M. C. S. Wong, W. Y. Cheng, and C. Y. P. Wong, "Market risk management of banks: implications from the accuracy of value-at-risk forecasts," Journal of Forecasting, vol. 22, no. 1, pp. 23-33, 2003.

[23] J.-B. Su and J.-C. Hung, "Empirical analysis of jump dynamics, heavy-tails and skewness on value-at-risk estimation," Economic Modelling, vol. 28, no. 3, pp. 1117-1130, 2011.

[24] C.-M. Kuan, J.-H. Yeh, and Y.-C. Hsu, "Assessing value at risk with CARE, the conditional autoregressive expectile models," Journal of Econometrics, vol. 150, no. 2, pp. 261-270, 2009. 